\title{
The influence of Laval nozzle throat size on supersonic molecular beam injection
}

\author{
Xinkui He $\cdot$ Xianfu Feng $\cdot$ Mingmin Zhong $\cdot$ \\ Fujun Gou $\cdot$ Shuiquan Deng $\cdot$ Yong Zhao
}

Received: 3 December 2013/Revised: 25 February 2014/ Accepted: 10 March 2014/Published online: 10 April 2014

(C) The Author(s) 2014. This article is published with open access at Springerlink.com

\begin{abstract}
In this study, finite element analysis (FEA) has been used to investigate the effects of different Laval nozzle throat sizes on supersonic molecular beam. The simulations indicate the Mach numbers of the molecular stream peak at different positions along the center axis of the beam, which correspond to local minimums of the molecular densities. With the increase of the throat diameter, the first peak of the Mach number increases first and then decreases, while that of the molecular number density increases gradually. Moreover, both first peaks shift progressively away from the throat. At the last part, we discuss the possible applications of our FEA approach to solve some crucial problems met in modern transportations.
\end{abstract}

Keywords Nuclear fusion - Molecular beam injection . Mach number - Laval nozzle throat size

\section{Introduction}

The technique of injecting fuel into the center of a nuclear fusion reactor or into a Tokamak device has remained a challenging problem in the realistic applications of nuclear

X. He $\cdot$ X. Feng $\cdot$ S. Deng $(\bowtie) \cdot$ Y. Zhao $(\bowtie)$

Key Laboratory of Magnetic Levitation Technologies and

Maglev Trains, Ministry of Education of China,

Chengdu 610064, China

e-mail: s.deng@swjtu.edu.cn

X. He $\cdot$ X. Feng $\cdot$ S. Deng $\cdot$ Y. Zhao

Superconductivity and New Energy R\&D Center, Southwest Jiao

Tong University, Chengdu 610064, China

M. Zhong · F. Gou

Institute of Nuclear Science and Technology,

Sichuan University, Chengdu 610064, China fusion energy. The confinement time and the density peak position of the Plasma are key performance parameters for a thermonuclear reactor or device [1,2]. It has been known that the density peak is tightly related to the injection method. The main existing injection methods include normal injection, ice pellet injection, and supersonic molecular beam injection. The normal injection method has the disadvantages of low efficiency and difficulty in forming the density peaks. Though the pellet injection method has many advantages, the relevant techniques for the whole injection system are rather complicated, which results in too expensive running costs [1,3-5]. The supersonic molecular beam injection as first proposed by Yao et al. [6] is a new method for injecting the fuel and has been successfully applied in HL-1 M, HT-7, EAST, and HL-2A of China [2, 3, 7-11]. This method has also been accepted as a candidate mode for fuel injection in the international thermonuclear fusion reactor by the relevant experts [9]. In this approach, the supersonic molecular beam is formed through the Laval nozzle throat by making use of the pressure difference [3]. In this work, we study the relation between the Laval nozzle throat size and the Mach numbers and the density peaks of the injected molecular beams. Our results agree well with those previously obtained by other methods [12, 13].

\section{Simulation details}

Figure 1 shows a sketch of our simulation setup composed of a Laval nozzle device and a vacuum chamber. In this setup, the length of the Laval nozzle and that of the vacuum chamber are chosen as 30 and $120 \mathrm{~mm}$, respectively. As shown in Fig. 1, the inlet/outlet diameter of the nozzle is 6 and $12 \mathrm{~mm}$, respectively. The distance from the throat to 


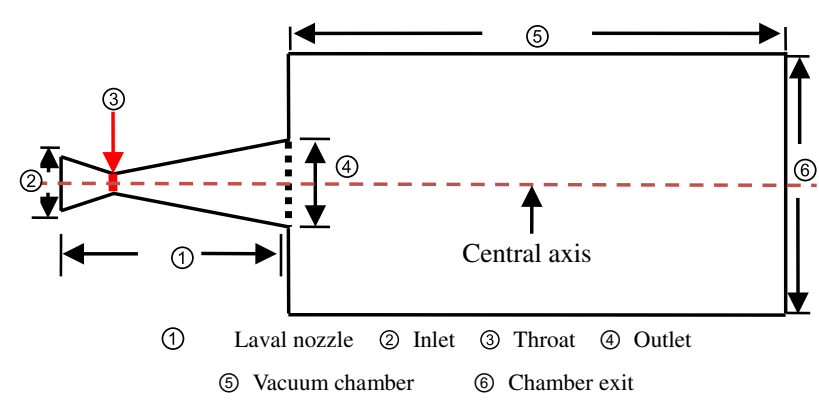

Fig. 1 Simulation model for the formation of the supersonic molecular beam

the inlet/outlet is 4 and $26 \mathrm{~mm}$, respectively. The width of the chamber is chosen as $40 \mathrm{~mm}$.

The high pressure gas passes through the Laval nozzle and finally expands into the vacuum chamber resulting in a supersonic molecular beam.

\subsection{Simulation principles}

In this work, the simulation is realized by using the finite element method as implemented in COMSOL Multiphysics software package. According to the characteristics of the neutral beam injection, the following assumptions have been made through the computational fluid dynamics (CFD) module of the above package:

(1) The molecular beam is assumed as a Newtonian fluid which satisfies the Navier-Stokes $(\mathrm{N}-\mathrm{S})$, the continuity, and energy conservation equations.

(2) The fluid is assumed to be a steady, axisymmetric, isotropic, and adiabatic laminar flow.

(3) The gas in the chamber is assumed to be in the ideal state.

(4) The chamber wall meets thermal insulation and noslip conditions.

(5) The thermal conductivity of the molecular beam satisfies the Fourier's Law: $\vec{q}=-K \nabla T$.

Based on the above assumptions, the physical process of the supersonic molecular beam can be simulated by solving the combined equations of momentum conservation $(\mathrm{N}-\mathrm{S})$, continuity, and energy conservation.

The nutshell of the problem is the $\mathrm{N}-\mathrm{S}$ equation as follows:

$\rho \frac{\partial \vec{u}}{\partial t}+\rho(\vec{u} \cdot \nabla) \vec{u}=\vec{F}-\nabla P+\mu \nabla^{2} \vec{u}+\frac{\mu}{3} \nabla(\nabla \cdot \vec{u})$.

The density and the velocity of the molecular beam satisfy further the following continuity equation $\nabla \cdot(\rho \vec{u})=0$

The whole process satisfies the energy conservation condition as indicated by the following equation:

$\rho c_{\mathrm{v}}(\vec{u} \cdot \nabla) T=K \nabla^{2} T-\rho(\nabla \cdot \vec{u}) T$.

In the chamber, the gas is assumed to satisfy the following ideal state equation,

$P=\rho R_{\mathrm{s}} T$.

It should be pointed out that Eq. (4) is an approximation to the actual situation because the gas in the chamber is obviously not in a steady state, neither is it in an ideal state. However, this approximation has also been used by other researchers in the related fields $[14,15]$.

By solving the above four main equations, one can obtain the velocity field in the system and thus find the Mach number by the following equation:

$M_{\mathrm{a}}=\frac{\vec{u}}{c} ; c=\sqrt{\gamma R T}$.

In the above equations, the symbol $\rho$ represents the mass density of the fluid, $\vec{u}$ the velocity field of fluid, $\vec{F}$ the external force per unit volume, $\mu$ the coefficient of viscosity, $C_{\mathrm{v}}$ the isochoric specific heat capacity, $T$ the temperature, $K$ the thermal conductivity, $c$ the sound velocity as defined in Eq. (5), $R_{\mathrm{S}}$ the specific gas constant, $R$ the gas constant as defined in the state equation of ideal gas, and $\gamma$ the ratio of constant-pressure heat capacity versus constant-volume heat capacity (1.412 for hydrogen), respectively.

For a steady, $\frac{\partial \vec{u}}{\partial t}=0$, adiabatic and isotropic fluid, Eq. (1) is simplified as follows:

$(\vec{u} \cdot \nabla) \vec{u}=\frac{1}{\rho} \vec{F}-\frac{1}{\rho} \nabla P+\frac{1}{\rho} \mu \nabla^{2} \vec{u}+\frac{\mu}{3} \frac{1}{\rho} \nabla(\nabla \cdot \vec{u})$.

The term $(\vec{u} \cdot \nabla) \vec{u}$ in Eq. (6) is called convective acceleration caused by the uneven distribution of the velocity field. The term $-\frac{1}{\rho} \nabla P$ denotes the force caused by pressure gradient, while $\frac{1}{\rho}\left(\frac{\mu}{3} \nabla(\nabla \cdot \vec{u})\right)$ and $\frac{1}{\rho} \mu \nabla^{2} \vec{u}$ indicate the forces originated from viscosity. Equation (3) is the first-order approximation of the energy conservation equation. The first term in the right side of Eq. (3) is due to the heat flux, while the second term is essentially the work done by pressure. It should be noted that the isentropic and other approximations as used in our simulations, on one hand, have simplified the actual rather complicated process of the gas jet through the Laval nozzle; however, they have unavoidably resulted in some deviations from the actual process [15]. The quantitative estimations for the consequences of such approximations are left for our future work. 
As shown in Fig. 1, our model is axis-symmetric with the center line of the Laval nozzle as the symmetry axis. This two-dimensional model is triangulated as in normal finite elemental calculations. Some important boundary conditions are listed in Table 1.

\section{Results}

As shown in Fig. 2, when the injected gas jet enters into the vacuum chamber, a shock wave is formed. Figure 2a shows the simulated images of the shock waves for different Laval throat sizes. Figure $2 b$ shows the throat-size dependence of the Mach numbers and their changes along the center axis. As shown in Fig. 2, when a high pressured gas is injected into the chamber through the Laval throat, a free jet is formed at the expansion stage and is confined in a sectional region. The free jet zone includes the quiet region $\left(M_{\mathrm{a}} \gg 1\right)$, barrel shock wave area $\left(M_{\mathrm{a}}>1\right)$ at the two sides of the center axis, and the Mach shock wave area $\left(M_{\mathrm{a}}<1\right)$.

As shown in Fig. 2b, multiple peaks of the Mach numbers occur along the center axis of the beam. In the region between the entrance and throat of the Laval nozzle, the Mach numbers increase gradually which have a weak dependence on the throat size. Passing through the throat, the Mach numbers increase rapidly and form a multi-peak structure. At a throat size of $1.2 \mathrm{~mm}$, the first peak of Mach numbers reaches a maximum value of 2.569. It can be seen that the peaks of the Mach numbers shift toward the chamber with the increases of the Laval nozzle throat diameters. As can be expected, the supersonic character indicated with the Mach numbers decays inside the depth of the chamber.

Figure 3a shows the distributions of molecular number densities along the center axis and their dependence on the throat diameters. As for the Mach numbers, the molecular number densities have very weak throat-size dependence in the region between the inlet and throat. The calculated number density is about $1.6 \times 10^{24} / \mathrm{m}^{3}$ in this region. From the throat into the chamber, a multi-peak structure is formed. For a throat diameter of $0.8 \mathrm{~mm}$, only two peaks are formed, while for the other three cases, i.e., 1.2, 1.6, and $2.0 \mathrm{~mm}$, three peaks are formed. The positions of the

Table 1 Boundary condition of simulation

\begin{tabular}{ll}
\hline Boundary & Condition \\
\hline Inlet & $P_{0}=5,000 \mathrm{~Pa} ; T=300 \mathrm{~K}$ \\
Fluid & $k=\mu C_{\mathrm{p}} / P_{\mathrm{r}} ; \mu=\mu_{\text {ref }}\left(T / T_{\text {ref }}\right)^{3 / 2}\left(T_{\text {ref }}+S\right) /(T+S)$ \\
Wall & $\vec{u}=0 ;-\vec{n} \cdot(-k \nabla T)=0$ \\
Outlet & $P_{\mathrm{b}}=1,000 \mathrm{~Pa}$ \\
\hline
\end{tabular}
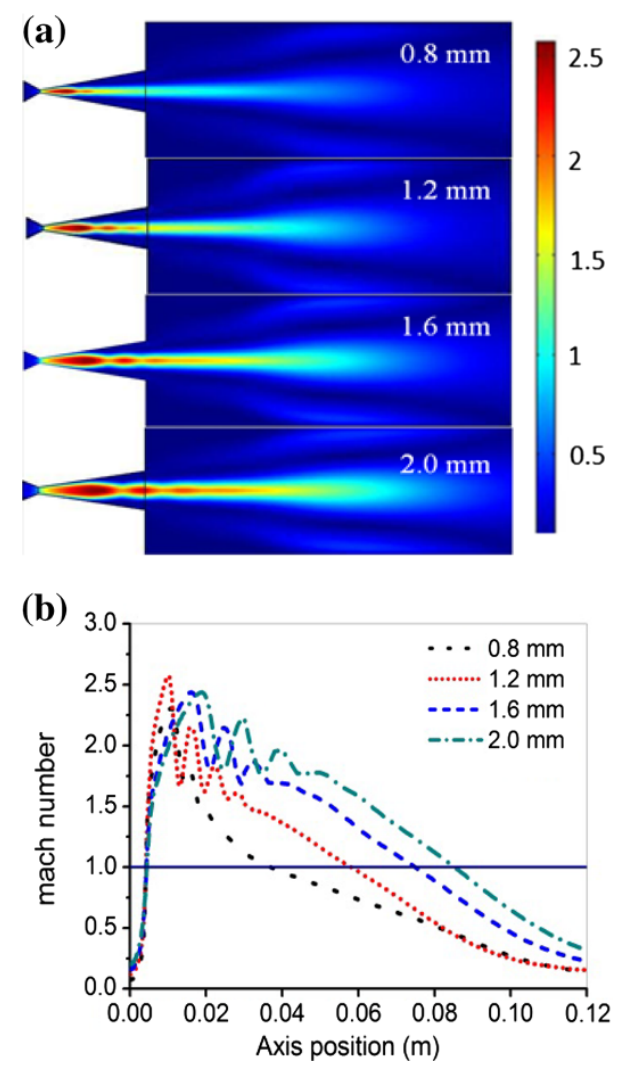

Fig. 2 a Simulated images of the supersonic molecular beams at different Laval nozzle throat diameters, the color bar measures the Mach numbers. b The distribution of the Mach numbers along the center axis and their dependence on the Laval nozzle throat size

peaks shift toward the inside of the chamber with the increase of the throat sizes. The molecular number densities reach an identical value of $0.27 \times 10^{24} / \mathrm{m}^{3}$ at the deep inside of the chamber. As a comparison, the simulated result of George [12] is also given in Fig. 3b. At a throat size of $1.2 \mathrm{~mm}$, the value of the first peak is $0.379 \times 10^{24} / \mathrm{m}^{3}$. Through comparing Fig. 2 with Fig. 3, one can find the relation between the occurrence of molecular density peaks and the occurrence Mach-number peaks.

\section{Discussions and conclusions}

Our simulations indicate that the Mach numbers on the center axis of the beam show a multi-peak structure as shown in Fig. 1b, which correspond to the local valleys of molecular number-density peaks. An optimum throat diameter of $1.2 \mathrm{~mm}$ is obtained as required by the efficiency conditions of the injection. It should be pointed out that the above results have been obtained by assuming the approximations listed in Sect. 2.1. Though these approximations have been used in the standard CFD module of COMSOL package, further studies are worthy to go beyond them to judge their validity in the current case. 

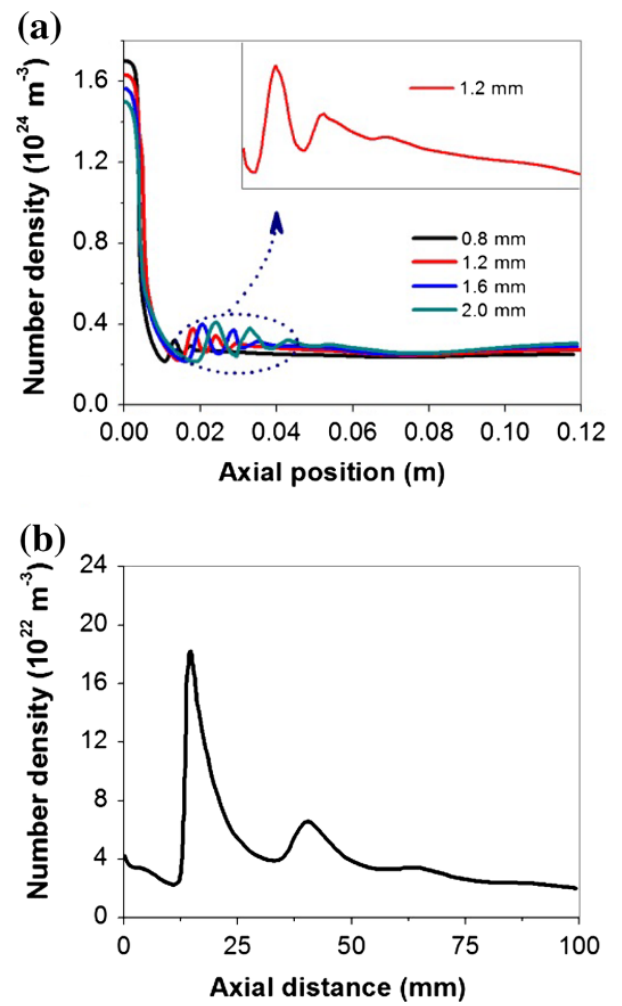

Fig. 3 a The distributions of Molecular number densities along the center axis and their dependence on the throat size; $\mathbf{b}$ The simulated result of George [12]

It should be noted that the aerodynamics of the highspeed train and in particular the maglev train or the other conceptual high-speed transportations can be similarly solved as in this work. The similar ANSYS CFD module has already been used to simulate the shear stress and aerodynamics of capsule in the conceptual hyperloop design $[16,17]$. In addition to the gas or fluid problem, the FEA method can also be used to simulate the rail steel microstructure and crack growth met in the complicated wheel-rail interactions [18].

Acknowledgments This work is financially supported by the Science Foundation for International Cooperation of Sichuan Province (2014HH0016), the Fundamental Research Funds for the Central Universities (SWJTU2014: A0920502051113-10000), National Magnetic Confinement Fusion Science Program (2011GB112001).

Open Access This article is distributed under the terms of the Creative Commons Attribution License which permits any use, distribution, and reproduction in any medium, provided the original author(s) and the source are credited.

\section{References}

1. Chen CY, Yao LH, Feng BB et al (2010) Study of SMBI fueling efficiency on HL-2A tokamak. Nucl Fusion Plasma Phys 30(1):1-6

2. Yao LH (1999) Supersonic molecular beam fueling in the tokamak. Physicas 28(2):113-116

3. Yao LH, Feng BB, Feng Z et al (2002) Effects of fueling by using high-pressure supersonic molecular beam in the HL-1 M tokamak. Acta Phys Sinica 51(3):596-602

4. Dong JF, Tang NY, Li W et al (2002) Diagnosis of the penetration characteristics for supersonic molecular beaminjection in the HL-1 M tokamak. Acta Phys Sinica 51(9):2074-2079

5. Jiao YM, Yao LH (2002) Optimization analysis of tokamak plasma fuelling with supersonic molecular beam. Nucl Fusion Plasma Phys 22(1):13-16

6. Yao LH, Tang YN, Ding XT (1998) A new fueling method for fusion plasma: supersonic molecular beam injection. Nucl Fusion Plasma Phys 18:91-97

7. Pan $\mathrm{CH}$ (2010) The international thermonuclear experimental reactor and the future of nuclear fusion energy. Physics 39(6): 375-378

8. Hu LQ, Zhang XD, Yao RH (2006) EAST neutral beam injection project for EAST Tokamak. Nucl Tech 29(2):149-152

9. Feng BB, Yao LH, Li W et al (2008) Observations of SMBI penetration depth in the HL-2A tokamak with divertor configuration. Nucl Fusion Plasma Phys 28(2):97-100

10. Soukhanovskii VA, Kugel HW, Kaita R et al (2004) Supersonic gas injector for fueling and diagnostic applications on the National Spherical Torus Experiment. Rev Sci Instrum 75(10): $4320-4324$

11. Soukhanovskii VA, Bell MG, Blanchard WR et al (2007) High pressure supersonic gas jet fueling on NSTX. IEEE 7(7):11-14

12. George JD (2000) A combined CFD-DSMC method for numerical simulation of nozzle plume flows. Dissertation, Cornell University, New York, USA

13. Richard III AN (2001) Navier stokes direct simulation Monte Carlo modeling of small cold gas thruster nozzle and plume flows. Dissertation, Worcester polytechnic Institute, Worcester, MA, USA, 2002

14. Gasser I, Rybicki M (2013) Modelling and simulation of gas dynamics in an exhaust pipe. Appl Math Model 37(5):2747-2764

15. Wang LP, Qiu AC, Kuai B et al (2005) Study of the gas-puff line mass and density from Laval nozzle. High Power Laser Part Beams 22(2):295-298 in Chinese

16. Alyssa A (2013) Hyperloop simulation shows it could work, Discovery News, http://news.discovery.com/tech/gear-and-gadgets/ hyperloop-simulation-shows-it-could-work-130920.htm. 20 Sep 2013

17. Statt N (2013) Simulation verdict: Elon Musk's hyperloop needs tweaking, CNET News, http://news.cnet.com/8301-11386_357602897-76/simulation-verdict-elon-musks-hyperloop-needstweaking/. 19 Sep 2013

18. Franklin FJ, Gahlot A, Fletcher DI, Garnham JE, Davis C (2011) Three-dimensional modelling of rail steel microstructure and crack growth. Wear 271:357-363 